EESTI NSV TEADUSTE AKADEEMIA TOIMETISED. XIII KOIDE FOOSIKA-MATEMAATIKA- JA TEHNIKATEADUSTE SEERIA. 1964, NR. 3

ИЗВЕСТИЯ АҚАДЕМИИ НАУК ЭСТОНСКОИ ССР. ТОМ ХIII СЕРИЯ ФИЗИКО-МАТЕМАТИЧЕСКИХ И ТЕХНИЧЕСКИХ-НАУК. 1964, № 3

\title{
СПЕКТРОФОТОМЕТР СУМЕРЕЧНОГО НЕБА
}

\section{В. ПЫЛДМАА}

Существенная роль среди косвенных методов исследования физических свойств стратосферы и мезосферы принадлежит сумеречному методу.

Общий очерк современного состояния вопроса дается в недавно опубликованной монографии Г. В. Розенберга [']. Там же приведены оригинальные результаты теории сумеречных явлений, позволяющие по непосредственным измерениям яркости сумеречного неба получить ценные сведения о строении атмосферы. Экспериментальный материал, относящийся к спектральной яркости сумеречного неба, довольно обширен, но подавляющее число измерений относится к определенным точкам солнечного вертикаля, например $\left.{ }^{2-6}\right]$. Лишь немногие измерения, например [ $\left.{ }^{4-6}\right]$, выполнены в абсолютных единицах.

В 1963 г. в Институте физики и астрономии АН ӘССР произвели спектрофотометрирование сумеречного неба. Целью измерений было получение распределения спектральных яркостей в абсолютных энергетических единицах при зенитных расстояниях Солнца Ђө от 90 до $100^{\circ}$. А также исследование некоторых оптических свойств атмосферы, как например, рассеяние лучистой энергии на высотах от 20 до $100 \mathrm{\kappa m}$.

Применявшийся спектрофотометр состоит из следующих частей:

1. приемная система,

2. усилитель фототока,

3. регистратор,

4. механизм автоматического поворота спектрофотометра по вертикали.

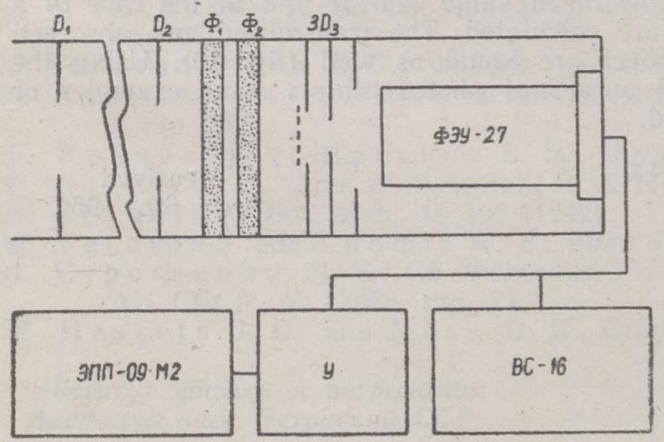

Принципиальная схема спектрофотометра изображена на рис. 1. Приемником радиации является фотоумножитель ФЭУ-27, помешенный в трубку с диафрагмами $D_{1}$ и $D_{3}$ для ограничения поля зрения фотометра. Диафрагма $D_{2}$ служит для избежания попадания отраженной радиации

Рис. 1. Принципиальна схема спектрофотометра. 
от стен трубки на приемник. Трубка снабжена закрывающим затвором 3 для определения нуля системы. $\Phi_{1}$ и $\Phi_{2}$ - оправы для интерференционных фильтров, для люминофора или нейтрального фильтра. В фотометре не применялось оптических систем.

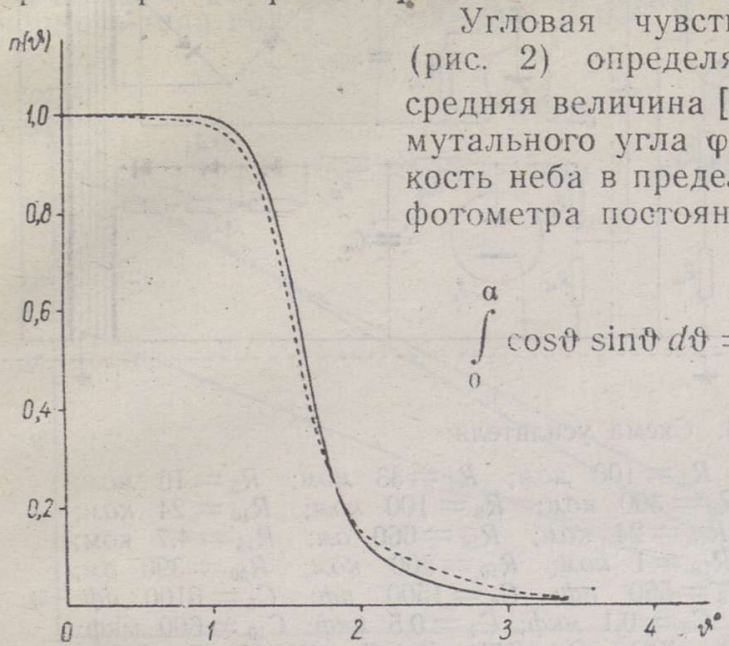

Рис. 2. Угловая чувствительность приемника $-\varphi,--\varphi+180^{\circ}$.

Выражение (1) дает

$$
a=\arcsin \left(2 \int_{0}^{\frac{\pi}{2}} \overline{n(\vartheta)} \sin \vartheta d \vartheta\right)^{\frac{1}{2}} .
$$

Соответственно эффективный угол зрения фотометра определяется выражением

$$
\text { (1) })_{\text {fff }}=2 \pi\left[1-\left(1-2 \int_{0}^{\frac{\pi}{2}} \overline{n(\vartheta)} \sin \vartheta d \vartheta\right)^{\frac{1}{2}}\right] \text {. }
$$

В данном случае $\omega_{\epsilon f f}=2,8 \cdot 10^{-3}$ стер.

Фототок ФЭУ-27 подается на усилитель, схема которого приведена на рис. 3. Девять пределов измерения позволяют изменять чувствительность системы всего в $7 \cdot 10^{3}$ раз. Усилитель имеет хорошую линейность во всех пределах измерения. Через 1,5 час после включения усилителя дрейф его нуля не превышает $0,2 \%$ от всей шкалы электронного потенциометра ЭПП-09M2 за час. Последний является регистратором усиленного фототока. Время пробега каретки всей шкалы применяемого потенциометра 1 сек.

Поворот приемника вокруг горизонтальной оси от горизонта до противоположного горизонта совершается автоматически при помощи азимутальной головки электрофотометра Главной геофизической обсерватории им. А. И. Воейкова [7]. После достижения горизонта затвор автоматически закрывается и записывается нуль системы.

Фотоумножитель питается от стабилизированного

выпрямителя ВC-16 напряжением $900 \div 1300$ в. 


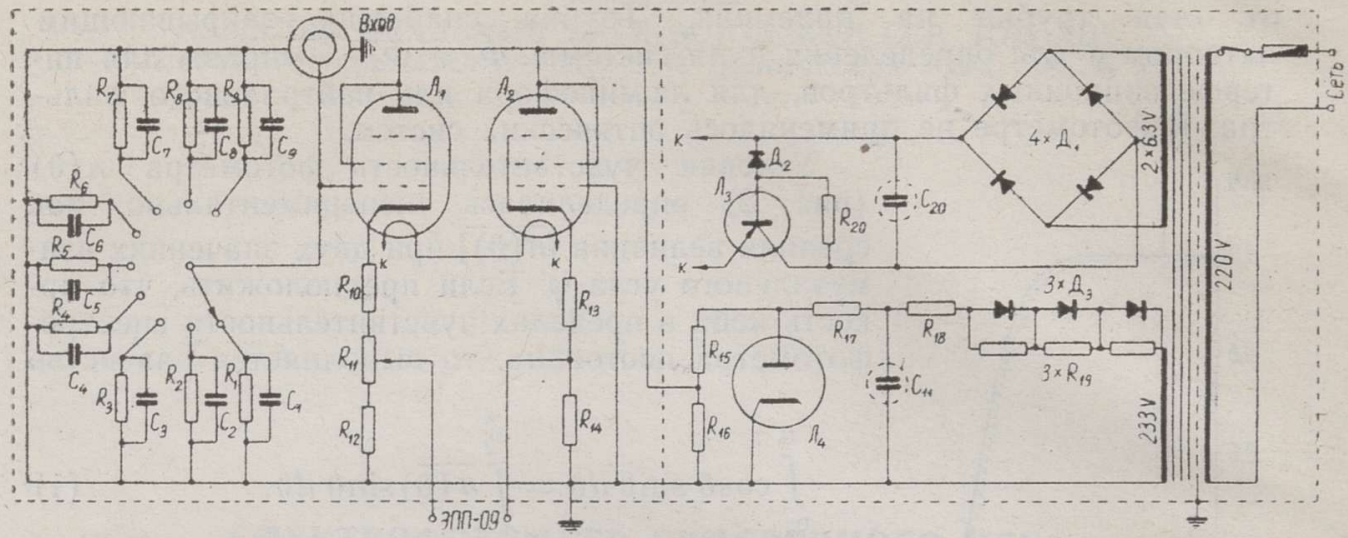

Рис. 3. Схема уснлителя:

$R_{1}=820$ мон; $R_{2}=330$ нон; $R_{3}=100$ мон; $R_{4}=33$ мом; $R_{5}=10$ ном; $R_{6}=3,3$ ном; $R_{7}=1$ мон; $R_{8}=300$ ком; $R_{9}=100$ кон; $R_{30}=24$ ком; $R_{11}=150 \mathrm{o \mu} ; \quad R_{12}=510 \mathrm{oн} ; \quad R_{13}=24 \mathrm{\kappa O \mu} ; \quad R_{14}=660$ он; $R_{15}=4,7$ ком; $R_{16}=6 \kappa$ ко; $R_{17}=5,8$ ком; $R_{18}=1$ кон; $R_{19}=200$ ком; $R_{20}=390$ ом; $C_{1}=60 n \phi ; \quad C_{2}=150 \quad n \phi ; \quad C_{3}=560 \quad n \phi ; \quad C_{4}=1500 \quad n \phi ; \quad C_{5}=6100 \quad n \phi ;$

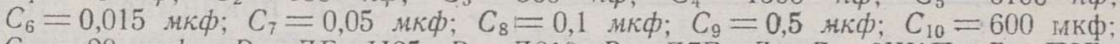
$C_{11}=20$ мкф; $D_{1}-Д Г-Ц 25 ; D_{2}-Д 810 ; D_{3}-$ Д $\pi_{4}-\mathrm{C} \Gamma 3 \mathrm{C}$.

Линейность всей измерительной системы определялась нейтральными фильтрами в монохроматическом потоке излучения. Результаты показывают, что нелинейность системы не превышает $0,5 \%$.

Чувствительность фотометра проверялась во время наблюдений через каждые $30-40$ мин путем измерения яркости люминофора постоянного действия. Предварительно было изучено влияние температуры на яркость люминофора. В рассматриваемом промежутке температур (от 0 до $25^{\circ} \mathrm{C}$ ) никаких изменений не было обнаружено. В течение всего пернода измерений (около 4 месяцев) регулярно сравнивалась яркость люминофора с яркостью лампы накаливания. Лампа, искусственно состаренная и расчитанная на питание 6 в, питалась напряжением $4,0300 \pm 0,0002$ в, измеряемом на цоколе лампы потенциометром постоянного тока Р375. Расхождение результатов сравнений не превышапо $\pm 1 \%$ и имело при этом несистематический характер.

Фотометр градуировался в абсолютных единицах энергии по прямой радиации Солнца методом, базирующимся на известной формуле Бугера. Для этого выбирали безоблачные дни с практически постоянной прозрачностью атмосферы. Критерием выполняемости этого требования служили измерения прямой радиации Солнца термоэлектрическим актинометром во время калибровки. Для ослабления потока лучистой энергии применялся нейтральный фильтр, спектральное пропускание которого определялось путем сравнивания его в монохроматическом потоке излучения с нейтральным фильтром НС-12 из комплекта цветного стекла. На рис. 4 изображены калибровочные кривые одного дня. На горизонтальной оси отложена масса атмосферы, на вертикальной - логарифм показания прибора. Калибровочные кривые экстраполировали до $m=0$ и сопоставили с данными прямой радиации Солнца за пределами атмосферы в соответствующих областях спектра по Джонсону [8].

Спектрофотометрирование проводилось в трех спектральных областях, выделенных при помоши интерференционных фильтров. Пропускание интерференционных фильтров определялось на спектрофотометре 
СФ-4. Там жеопределялась спектральная чувствительность ФЭУ -27 $k(\lambda)$. прнчем в качестве неселективного приемника был использован вакуум ный термоэлемент с окном из $\mathrm{KBr}$. Функция $k(\lambda)$ изображена на рис. 5 Эффективные длины волны, вычисленные для излучения абсолютно черного тела при $T^{\prime}=5400^{\circ} \mathrm{K}$ и $10000^{\circ} \mathrm{K}$, были следующие:

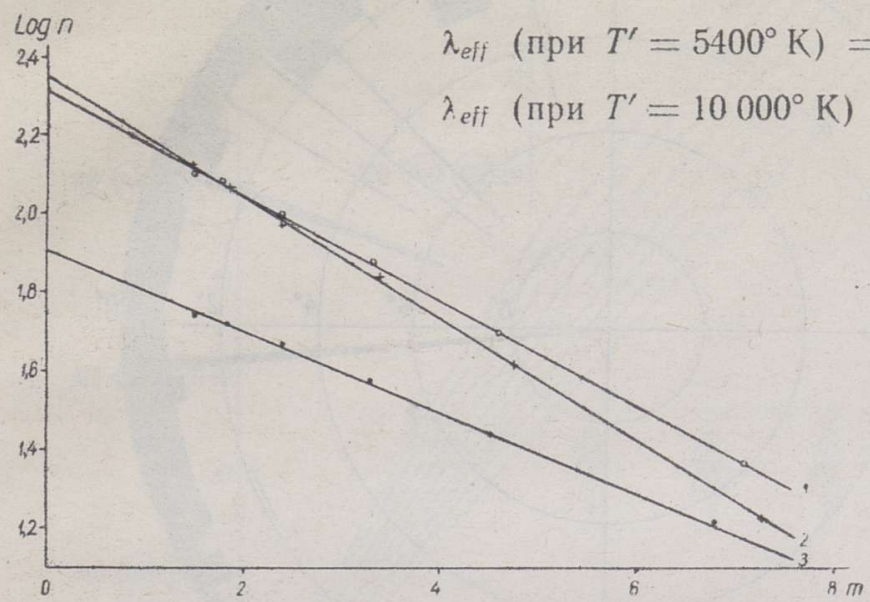

Рнс. 4. Прнмер градунровочных крнвых: $1-\lambda=479$ Анк; $2-\lambda=422$ нжк; $3-\lambda=574$ ик.

Описанный спектрофотометр применялся для определе ния яркостей сумеречного неба вблизи г. Тарту на плоцадке актинометрической станции Института физики и астрономии. Закрытость горизонта места наблюдений изображена на рис. 6. Высота места наблюдений $80 M$ над уровнем моря.

Измерения осуществиялись непрерывной записью в плоскости вертикали и противовертикали Солнца, а гакже в перпендикулярной им плоскости. Во время каждых сумерек применялись два интерференционных фильтра. Измерения пронзводились попеременно с фильтрами $\lambda_{\text {eff }}=422$ и 479 ммк или $\lambda_{\text {eff }}=422$ и 574 ммк. Для непрерывного прохождения от горизонта вертикала Солнца до горизонта противовертикала Солнца требовалось 45 сек. Таким образом, распределение яркостей в данной области спектра получилось в среднем через каждые две минуты. Через каждые 15-20 мин обоими фильтрами опреде-

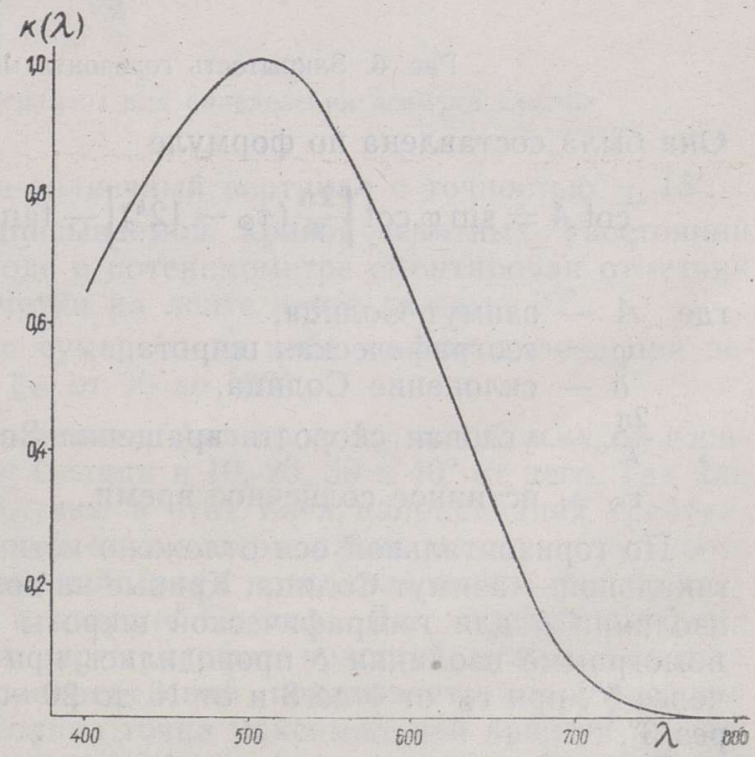

Рис. 5. Спектральная чувствительность ФЭУ-27. лялось распределение яркостей в плоскости, перпендикулярной вертикали Солнца.

Азимут Солнца для каждого момента времени определялся по соответствующей номограмме. Общий вид номограммы показан на рис. 7. 


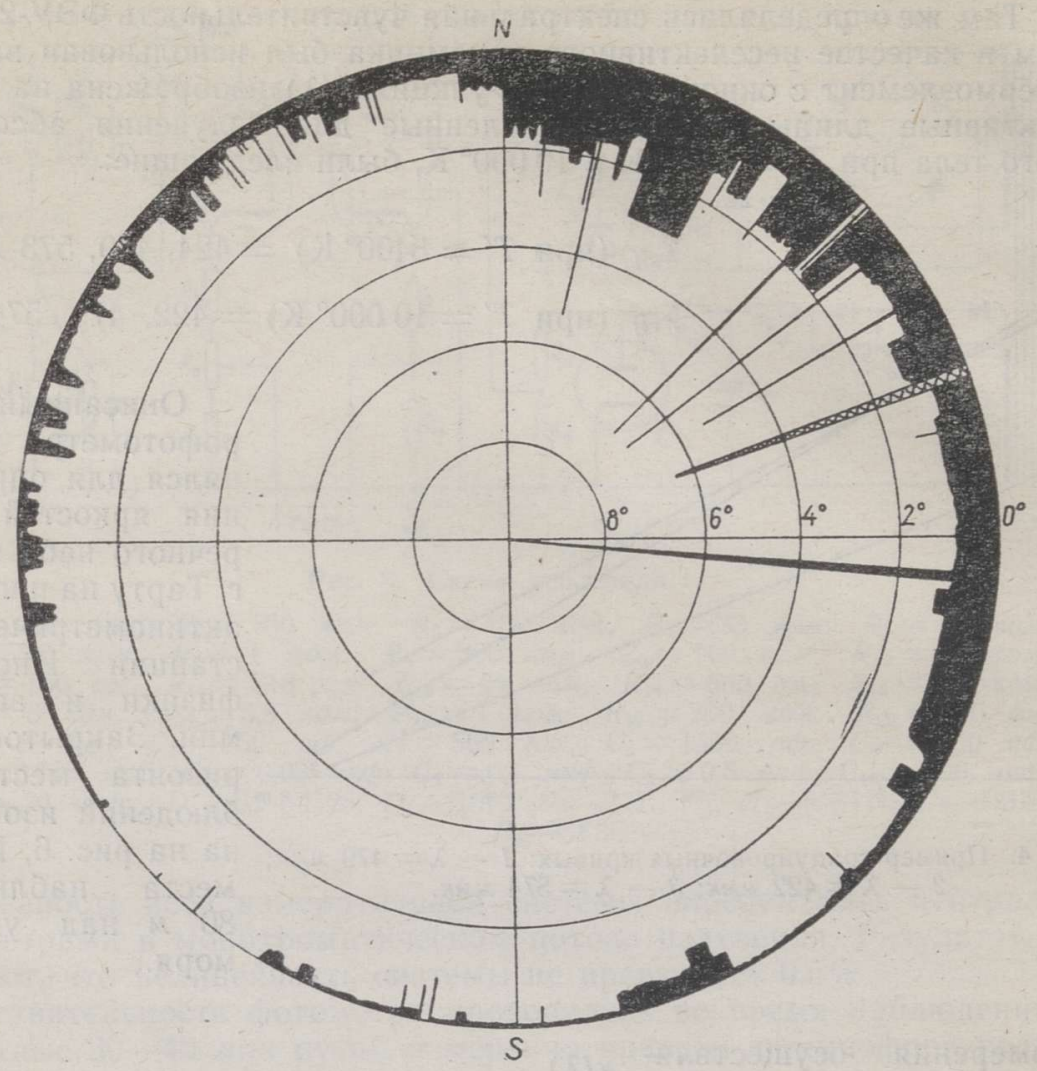

Рис. 6. Закрытость горизонта места наблюдений.

Она была составлена по формуле

$$
\cot A=\sin \varphi \cot \left[\frac{2 \pi}{T}\left(\tau_{\odot}-12^{h}\right)\right]-\tan \delta \frac{\cos \varphi}{\sin \left[\frac{2 \pi}{T}\left(\tau_{\odot}-12^{h}\right)\right]}
$$

где $A$ - азимут Солнца,

$\varphi$ - географическая широта,

о - склонение Солнца,

$\frac{2 \pi}{T}$

- угловая скорость вращения Земли,

$\tau_{\odot}$ - истинное солнечное время.

По горизонтальной оси отложено истинное солнечное время, по вертикальной - азимут Солнца. Кривые на номограмме представляют собой изолинии $\delta$ для географической широты г. Тарту. На использованной номограмме изолинии $\delta$ проводились при $\tau$ ॰ от 23 до 1 и от 11. до 13 через $6^{\circ}$, при $\tau_{\odot}$ от 4 до 8 и от 16 до 20 через $1^{\circ}$, в остальной части через $3^{\circ}$.

При определении значений $A$ по номограмме надо учитывать, что если соответствующие значения нанесены цифрами в кружках, то $\delta$ следует взять с противоположным знаком, причем значения $A$ нанесены тоже цифрами в кружках. На правом конце оси абсцисс имеется дополнительная часть значений $\tau$. Ею нужно воспользоваться в случае, если определенне $A$ для этих т॰ по основной части оси абсцисс оказывается невозможным, например, если $\tau \odot=17,30, \delta=+21^{n}$. 


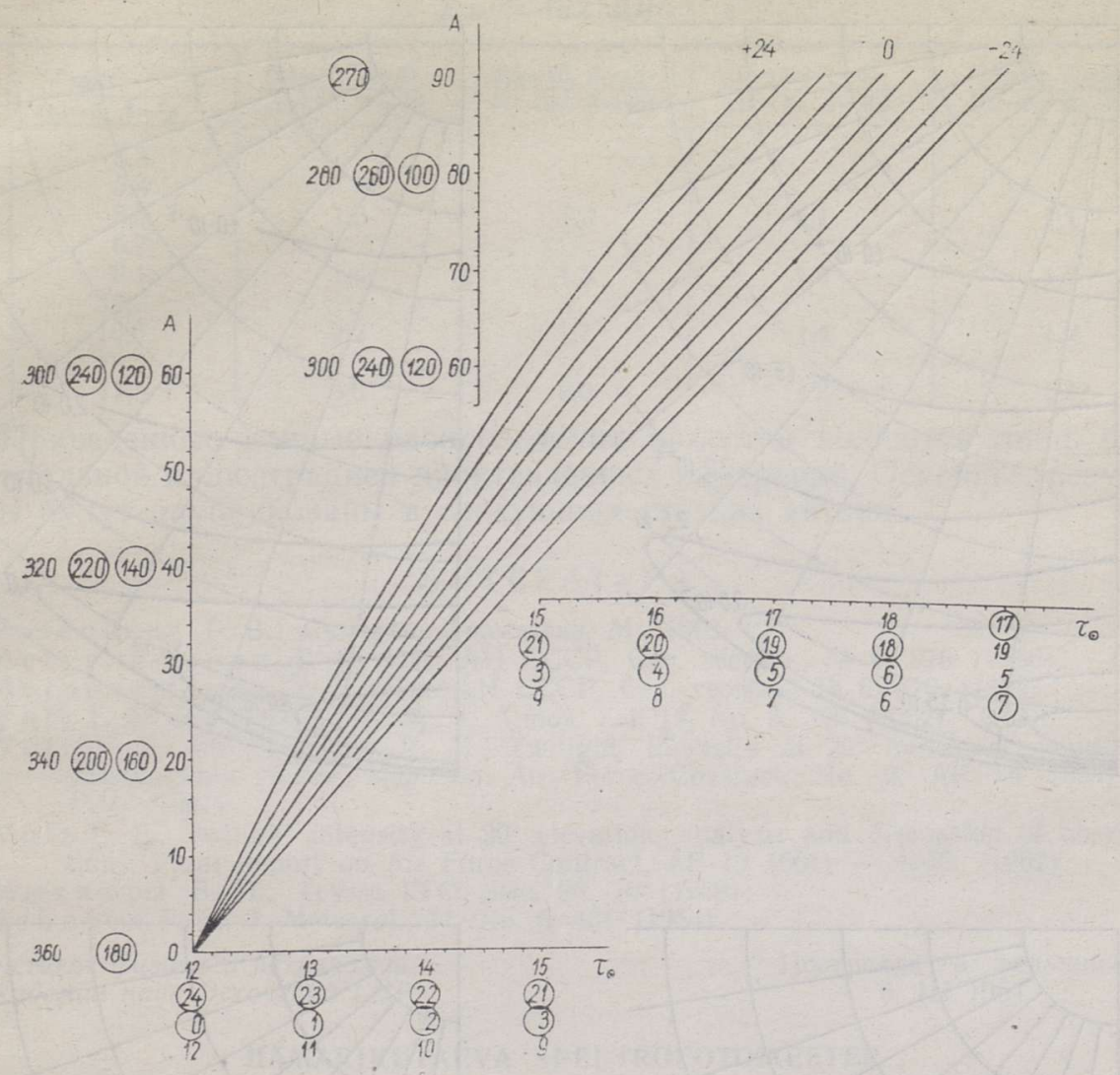

Рис. 7. Общий вид номограммы для определения азимута Солнца.

Фотометр нацеливался на солнечный вертикал с точностью $15^{\prime}$.

Для фиксирования на записываемой кривой зенитных ғасстояний измеряемых точек на небосводе в потенциометре смонтирован отметчик реперных точек, дающий отметки на ленте через каждые $10^{\circ}$.

Спектрофотометрирование сумеречного неба производилось при зенитных расстояниях Солнца Ђ॰ от 90 до $100^{\circ}$.

Были сделаны также измерения с фильтром $\lambda_{\text {eff }}=422$ ммк от горизонта до зенита по вертикалу Солнца и $10,20,30$ и $40^{\circ}$. от него. Так как для получения яркостной картины в этих пяти направлениях требовалось в среднем 4 мин, то графическая интерполяция между отдельными моментами времени и точками небосвода позволила найти распределение яркостей на небосводе в стороне Солнца. Для иллюстраций на

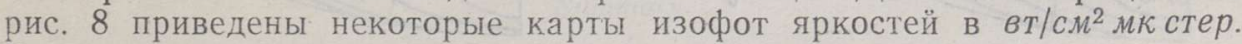
Рассмотрение полученных распределений показывает, что с увеличением зенитного расстояния Солнца точка максимальной яркости на вертикали Солнца становится все светлее относительно зенита. Сначала (до $\zeta_{\odot} \approx 94^{\circ}$ ) этот процесс происходит довольно медленно. При значениях ६॰ приблизительно с 94 до $98-99^{\circ}$ отношение $I\left(z_{\max }, \quad A=0\right) / I(z=0$, $A=0)$ быстро растет, затем опять замедляется и при ${ }_{\odot}$ около $100^{\circ}$ можно заметить противоположный ход.

Если сравнить яркость в точках небосвода с определенным зенитным расстоянием при $A=0$ и $A=40^{\circ}$, то выясняется, что для бо́льших $z$ 

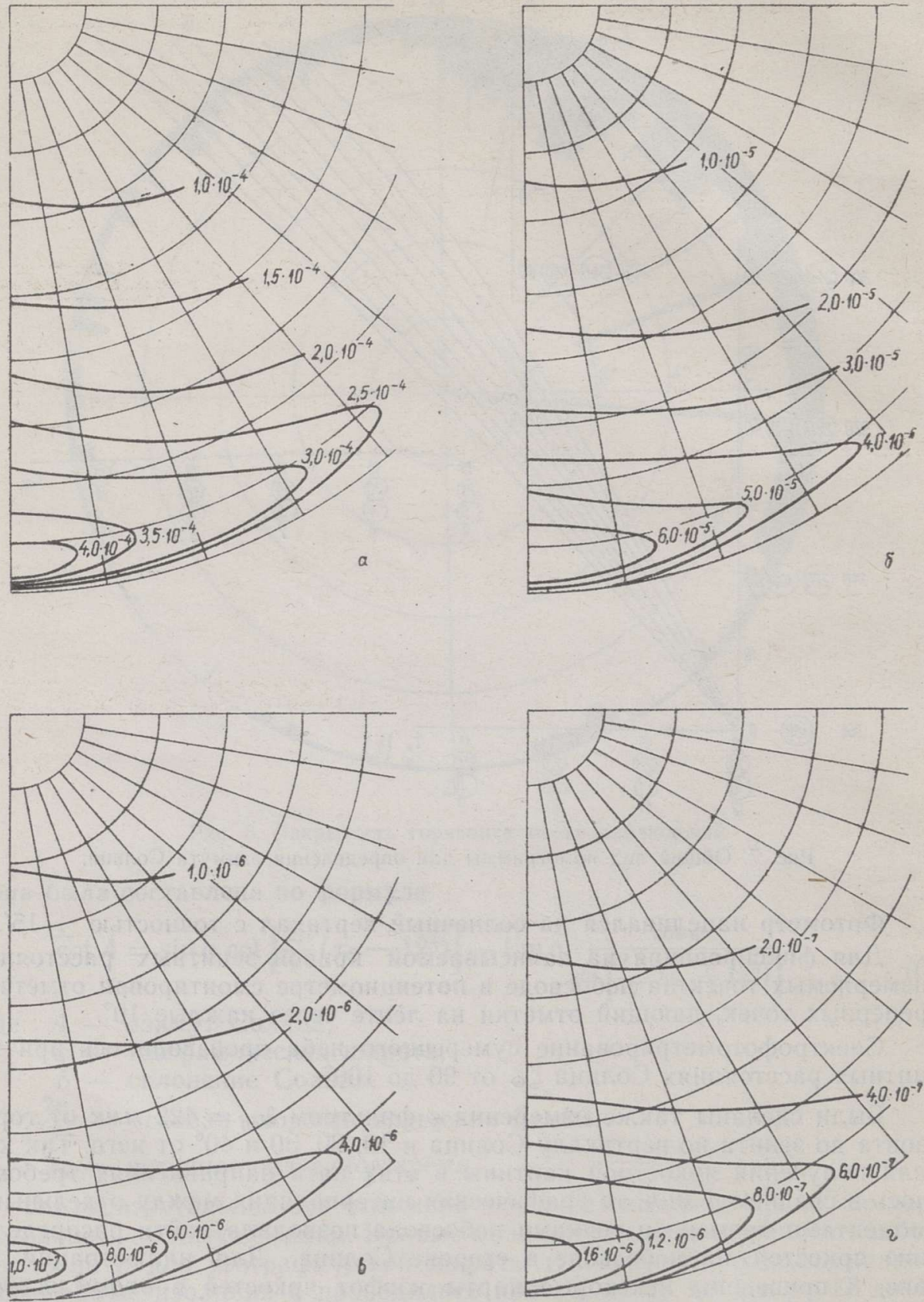

Рис. 8. Нзофоты яркостей $(\lambda=422$ мик) при: $a-\xi_{\odot}=94^{\circ} ; \sigma-\xi_{\odot}=96^{\circ} ; в-\zeta_{\odot}=98^{\circ} ; 2-\zeta_{\odot}=100^{\circ}$.

отношение $I\left(z_{0}, A=0\right) / I\left(z_{0}, A=40\right)$ растет с увеличением $\zeta$, для точек, расположенных ближе к зениту, оно проходит максимум при некотором значении Ђө. Для характеристики вышесказанного приводятся в таблице некоторые отношения. 


$$
\lambda_{\text {eff }}=422 \mu \kappa \kappa
$$

\begin{tabular}{r|c|c|c|c|c}
\hline$\zeta$ & $\frac{I\left(\max ^{\prime} A=0\right)}{I(z=0, A=0)}$ & $\frac{I(z=80, A=0)}{I(z=80, A=40)}$ & $\frac{I(z-60, A=0)}{I(z=60, A=40)}$ & $\frac{I(z=40, A=0)}{I(z=40, A=40)}$ & $\frac{I(z=20, A=0)}{I(z=20, A=40)}$ \\
\hline 92 & 5,3 & & & & \\
93 & 5,4 & 1,5 & 1,3 & 1,1 & 1,1 \\
94 & 5,5 & 1,5 & 1,5 & 1,3 & 1,2 \\
95 & 6,7 & 1,7 & 1,4 & 1,2 \\
96 & 9,1 & 2,2 & 2,0 & 1,2 & 1,1 \\
97 & 12,7 & 16,6 & & & \\
98 & 18,5 & 3,6 & 2,0 &
\end{tabular}

Приведенные данные распределения яркостей являются лишь первоначальной иллюстрацией произведенных измерений. Основные результаты будут опубликованы в следующих статьях автора.

\section{Л И Т Е Р А Т У Р А}

1. Р оз енбе рг Г. В., Сумерки, Физматгиз, М., 1963.

2. Мегр елишвили Т. Г., Изв. АН СССР, Сер. геофиз., № 8, 976 (1956).

3. М ег ре лиш ви ли Т. Г., Изв. АН СССР, Сер. геофиз., № 6, 910 (1959).

4. VoIz F. E. and Good y R. M., J. Atmos. Sci, 19, No. 5, 385 (1962).

5. Volz F. E. and Goody R. M., Twilight intensity at $20^{\circ}$ elevation, results of observations, Scient. Rep. on Air Force Contract, No. 2, AF 19 (604) 4546 (1961).

6. Volz F. E., Twilight intensity at $20^{\circ}$ elevation, analysis and discussion of observations, Final Report on Air Force Contract, AF 19 (604) - 4546 (1962).

7. Мюхкюр я В. И., Труды ГГО, вып. 93, 70 (1959).

8. Johns on F. S., J. Meteorol., 11, No. 6, 431 (1954).
Ннститут физики и астрономии
Академии начк Эстонской ССР
Поступила в редакщию
6. III 1964

\section{HAMMARIKUTAEVA SPEKTROFOTOMEETER}

\section{Põldmaa}

\section{Resümee}

Artiklis kirjeldatakse spektrofotomeetrit, mida kasutati hämarikutaeva heleduste jaotuse määramiseks Päikese vertikaalil ja temaga risti olevas tasandis. Kiirguse vastuvõtjana kasutati fotakordistajat $\Phi \ni У-27$. Fotovool võimendati eelvõimendajaga ja registreeriti elektronpotentsiomeetriga ЭПП-09M2. Fotomeeter kaliibriti absoluutsetes energeetilistes ühikutes. Mõõtmised teostati kolmes spektraalpiirkonnas: $\lambda_{\text {eff }}=422,479$ ja $574 \mathrm{~m} \mu$ Päikese seniitkaugustel $\zeta_{\odot}=90-100^{\circ}$. Esitatakse heleduste jaotus taevasfääri päikesepoolsel osal spektraalpiirkonnas $\lambda_{\text {eff }}=422 \mathrm{~m} \mu$ Päikese seniitkaugustel $\zeta_{\odot}=94,96,98 \mathrm{ja} 100^{\circ}$.

Eesti NSV Teaduste Akadeemia

Fü̈̈sika ja Astronoomia Instituut
Saabus toimetusse

6. III 1964

\section{TWILIGHT SKY SPECTROPHOTOMETER}

\section{Põldmaa}

\section{Summary}

A spectrophotometer used for measuring the brightness of twilight sky on the Sun's vertical and perpendicularly to it is described. The detector used was a photomultiplier $Ф Э У-27$. The photocurrent was amplified by a preamplifier and recorded by a self-compensating potentiometer absolute units of energy. The brightness was measured in spectral ranges $\lambda_{\text {eff }}=422,479$ and $574 \mathrm{~m} \mu$, when the zenith distance of the Sun was $\zeta_{\odot}=90-100^{\circ}$. The distribution of the sky brightness on the Sun's side of the hemisphere is given in the spectral range $\lambda_{\text {eff }}=422 \mathrm{~m} \mu$ by $\zeta_{\odot}=94,96,98,100^{\circ}$ 